\title{
Effect of Polymerization Conditions on Thermal and Mechanical Properties of Ethylene/1-Butene Copolymer Made with Ziegler-Natta Catalysts
}

\author{
Mostafizur Rahaman, ${ }^{1}$ M. Anwar Parvez, ${ }^{1}$ J. B. P. Soares, ${ }^{2}$ and I. A. Hussein ${ }^{1}$ \\ ${ }^{1}$ Department of Chemical Engineering, King Fahd University of Petroleum and Minerals, Dhahran 31261, Saudi Arabia \\ ${ }^{2}$ Department of Chemical and Materials Engineering, University of Alberta, Edmonton, AB, Canada T6G 2V4
}

Correspondence should be addressed to I. A. Hussein; ihussein@kfupm.edu.sa

Received 20 February 2014; Accepted 22 May 2014; Published 11 June 2014

Academic Editor: Giridhar Madras

Copyright ( 2014 Mostafizur Rahaman et al. This is an open access article distributed under the Creative Commons Attribution License, which permits unrestricted use, distribution, and reproduction in any medium, provided the original work is properly cited.

\begin{abstract}
The effect of polymerization conditions on thermal and mechanical properties of ethylene/1-butene copolymers synthesized through titanium-magnesium-supported Ziegler-Natta catalysts was studied. The increase in hydrogen pressure leads to a decrease in molecular weight (MW), storage modulus, and melting temperature. However, it yields an increase in molecular weight distribution (MWD), $\tan \delta, \%$ crystallinity, tensile modulus, yield stress, and strain at break. The effects of ethylene pressure and polymerization temperature on the copolymer MW, MWD and thermal and mechanical properties have been investigated. However, the impacts of ethylene pressure and polymerization temperature on copolymer modulus, tensile strength, \% crystallinity, crystallization peak temperature, yield stress, strain at break, and yield strain are marginal. The hydrogen pressure plays a major role in controlling the copolymer properties because it acts as an efficient chain transfer agent during polymerization reaction. The MW is the key parameter that influences flow activation energy. However, the other mechanical, dynamic mechanical, and thermal properties not only depend on MW but are also influenced by other parameters.
\end{abstract}

\section{Introduction}

The synthesis of ethylene-butene copolymers has been an attractive subject to many authors because of their interesting properties like low density, high degree of branching, and low cost price per unit volume compared to ethylene-propylene copolymer [1-3]. One of the great advantages of these copolymers is that they can also be reused without decreasing their processing and physical properties. Quan et al. have synthesized ethylene-butene copolymer through gas phase polymerization technique, where highly active catalysts like $\mathrm{TiCl}_{4}, \mathrm{SiO}_{2}$ or $\mathrm{ZnCl}_{2} /$ alcohol $/ \mathrm{AlR}_{3}$, and $\mathrm{Ti}(\mathrm{OBu})_{4} / \mathrm{MgCl}_{2}$ are used [4]. They have reported that the synthetic products have the properties of both oligomer and copolymer, and the melting temperature, crystallinity, and crystallite size decrease with the increase in butene content in the copolymer. The effect of polymerization conditions, like hydrogen and comonomer concentration, and polymerization time on the properties of ethylene-butene copolymer synthesized in two-step process has been reported elsewhere [1]. The influence of reaction time on ethylene/1-butene copolymer synthesized over supported titanium-magnesium catalyst has also been studied $[5,6]$. It has been shown that the increase in reaction time from 5 to $40 \mathrm{~min}$ results in small increase in molecular weight (MW), narrowing of molecular weight distribution (MWD), and decrease in 1-butene content in the copolymer.

Earlier, the synthesis of homo- and copolymer of ethylene using Zeigler-Natta and other different catalysts has been carried out where discussions have been made on various aspects of polymerization processes like the effect of catalyst type on copolymer properties, relationship of catalyst composition with molecular structure of homo- and copolymers, and so forth [7-10]. The advantages of using Zeigler-Natta catalysts are that they produce polymer products with high melting point, high MW, and controllable morphology. However, 


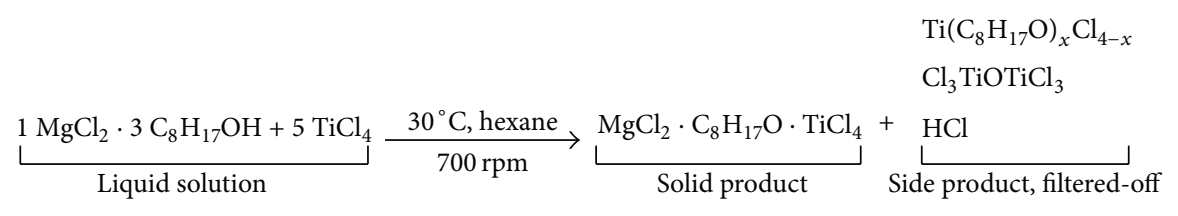

SCHEME 1: Synthesis of the magnesium-supported Ziegler-Natta catalyst.

there are some disadvantages associated with Ziegler-Natta catalysts. These catalysts have less control over the growing polymer chain branching because there is the presence of multiple metal sites on transition metal, and also the removal of catalyst from the final product is very difficult [11]. Recently, we have systematically discussed the effect of different polymerization conditions on dynamic mechanical, thermal, and mechanical properties of polyethylene homopolymers made with Ziegler-Natta catalysts [12]. Moreover, some correlations of homopolymers MW with different solid state properties were also made in the same literature [12].

In the present section, we have extended our previous work, where ethylene/1-butene copolymers have been synthesized over the same titanium-magnesium catalyst in laboratory-scale reactor and investigated the effect of different polymerization conditions on copolymers MW, MWD, thermal, and mechanical properties. Similarly, some correlations of copolymers MW with their solid state properties like dynamic mechanical, thermal, and tensile properties have been established and the fittings have been logically discussed.

\section{Materials, Methods, and Experimental Techniques}

2.1. Materials. Ultrahigh purity nitrogen (99.999\%) and polymer grade ethylene (99.9\%) were supplied by Praxair, Canada. All the operations were performed under nitrogen using standard Schlenk techniques or inside the glove box. Ethylene and nitrogen were purified by passing through columns. These columns are packed with R3-11 copper catalyst, activated alumina, and 3A/4A mixed molecular sieves. The materials such as titanium (IV) chloride $\left(\mathrm{TiCl}_{4}\right.$, $99 \%$ ) and magnesium chloride $\left(\mathrm{MgCl}_{2}\right.$, power-325 mesh) were procured from Aldrich (Canada) and 2-ethyl-1-hexanol (99\%) was purchased from Alfa Aesar, Germany. These materials were used for catalyst synthesis without further purification. Triethyl aluminum (TEA, $1 \mathrm{M}$ in hexane) was used as an activator and purchased from Aldrich, Canada. The reaction diluent, hexane (HPLC grade, 95\% n-hexane), was purchased from J. T. Baker, USA. This was used for the synthesis of catalyst and copolymer. It was purified by passing through columns packed with activated alumina and molecular sieves (Zeolum Type F-9, Tosoh Co., Japan). The purified solvent was stored in Schlenk flasks (Sigma-Aldrich, Canada) with 3A/4A mixed molecular sieves.
2.2. Synthesis of Magnesium-Supported Ziegler-Natta Catalyst. The solvent, hexane $(200 \mathrm{~mL})$, was taken to a $500 \mathrm{~mL}$ round bottled flask. Magnesium chloride $(7.62 \mathrm{~g}, 0.08 \mathrm{~mol})$ and 2-ethyl-1-hexanol $(37.5 \mathrm{~mL}, 0.24 \mathrm{~mol})$ were added to it. A clear solution of the mixture was obtained after stirring and refluxing for 24 hours. Titanium (IV) chloride $(0.5 \mathrm{~mol})$ was slowly added dropwise to it at a stirring rate of $700 \mathrm{rpm}$ and at a temperature of $30^{\circ} \mathrm{C}$. The mixture was kept for 2 hours to complete the reaction. The obtained precipitate was filtered and washed three times with hexane followed by drying under nitrogen flow. Finally, at the end of reaction, a dried white powder was isolated. Scheme 1 represents the synthetic route for the synthesis of the catalyst.

2.3. Synthesis of Ethylene/1-Butene Copolymers. The synthesis of ethylene/1-butene copolymers is conducted under various reaction conditions using magnesium-supported ZieglerNatta catalyst. A semibatch autoclave reactor $(300 \mathrm{~mL})$ was used for the polymerization reactions. This reactor was composed of a mass flow meter and a temperature control unit. The temperature was controlled with a cooling coil and an electric heater. The tolerance of polymerization temperature was $\pm 0.2^{\circ} \mathrm{C}$ of the set point. The reactor was purged five times with nitrogen before each reaction followed by heating up to $140^{\circ} \mathrm{C}$ under vacuum. The reactor was purged again to the set point temperature under nitrogen flow. Solvent $(200 \mathrm{~mL})$ and triethyl aluminum activator $(2.0 \mathrm{mmol})$ were taken in to the reactor followed by stirring for 5 minutes. Catalyst slurry $(3.0 \mathrm{mg})$ mixed with hexane was injected into the reactor and was stirred for 10 minutes. In this typical polymerization process, as, for example, the first run for sample 1, ethylene was continuously flowed to meet the ethylene pressure of 5 bar inside the reactor under a stirring rate of $500 \mathrm{rpm}$. Prior to ethylene, 1-butene $(71.3 \mathrm{mmol})$ was fed into the reactor. Hydrogen gas was passed to the reactor until the pressure reached 1 bar. The polymerization/reaction temperature was adjusted to $60^{\circ} \mathrm{C}$ and the polymerization was carried out for 30 mins. Ethylene flow to the reactor was stopped at the end of the polymerization time and the reactor was rapidly vented for reducing pressure and removing reactant. The set temperature was reduced to room temperature. The copolymer so obtained was precipitated in $200 \mathrm{~mL}$ ethanol followed by filtering and finally drying under vacuum. The detailed reaction condition and composition for all the copolymer samples are given in Table 1.

2.4. Gel Permeation Chromatography (GPC). Gel permeation chromatography (GPC-IR, Polymer Char, Spain) was 
TABLE 1: Ethylene/1-butene copolymerization data (polymerization time: $30 \mathrm{~min}$ ).

\begin{tabular}{|c|c|c|c|c|c|c|c|}
\hline s/number & $P_{\mathrm{C}_{2} \mathrm{H}_{4}}($ bar $)$ & 1-Butene fraction $(\mathrm{mmol})$ & $\begin{array}{c}\text { Poly. } \\
\text { temp. }\left({ }^{\circ} \mathrm{C}\right)\end{array}$ & $P_{\mathrm{H}_{2}}($ bar $)$ & MW (g/mol) & $\mathrm{Mn}(\mathrm{g} / \mathrm{mol})$ & PDI (MWD) \\
\hline 1 & 5 & 71.3 & 60 & 1 & 162534 & 55472 & 2.93 \\
\hline 2 & 5 & 71.3 & 60 & 2 & 149653 & 45765 & 3.27 \\
\hline 3 & 5 & 71.3 & 60 & 3 & 123342 & 31871 & 3.87 \\
\hline 4 & 5 & 71.3 & 80 & 1 & 132669 & 42117 & 3.15 \\
\hline 5 & 5 & 71.3 & 80 & 2 & 119172 & 34442 & 3.46 \\
\hline 6 & 5 & 71.3 & 80 & 3 & 107637 & 26775 & 4.02 \\
\hline 7 & 5 & 142.6 & 60 & 1 & 144653 & 42978 & 3.53 \\
\hline 8 & 5 & 142.6 & 60 & 2 & 130350 & 33337 & 3.91 \\
\hline 9 & 5 & 142.6 & 60 & 3 & 121622 & 26967 & 4.51 \\
\hline 10 & 5 & 142.6 & 80 & 1 & 129823 & 34163 & 3.80 \\
\hline 11 & 5 & 142.6 & 80 & 2 & 101153 & 23800 & 4.25 \\
\hline 12 & 5 & 142.6 & 80 & 3 & 91842 & 19335 & 4.75 \\
\hline 13 & 10 & 71.3 & 60 & 1 & 176583 & 61254 & 2.88 \\
\hline 14 & 10 & 71.3 & 60 & 2 & 159136 & 51734 & 3.08 \\
\hline 15 & 10 & 71.3 & 60 & 3 & 131776 & 36043 & 3.66 \\
\hline 16 & 10 & 71.3 & 80 & 1 & 141970 & 46828 & 3.03 \\
\hline 17 & 10 & 71.3 & 80 & 2 & 123435 & 37772 & 3.27 \\
\hline 18 & 10 & 71.3 & 80 & 3 & 112062 & 29464 & 3.80 \\
\hline 19 & 10 & 142.6 & 60 & 1 & 156754 & 45182 & 3.47 \\
\hline 20 & 10 & 142.6 & 60 & 2 & 142238 & 36729 & 3.87 \\
\hline 21 & 10 & 142.6 & 60 & 3 & 126835 & 29251 & 4.34 \\
\hline 22 & 10 & 142.6 & 80 & 1 & 135454 & 37635 & 3.60 \\
\hline 23 & 10 & 142.6 & 80 & 2 & 116068 & 28714 & 4.04 \\
\hline 24 & 10 & 142.6 & 80 & 3 & 96752 & 21543 & 4.49 \\
\hline
\end{tabular}

used to measure molecular weight and molecular weight distribution of copolymers. The samples were dissolved in $1,2,4$-trichlorobenzene at $160^{\circ} \mathrm{C}$ followed by passing through three linear Polymer Laboratories columns. These columns were calibrated with polystyrene standards and operated at a flow rate of $1 \mathrm{~mL} / \mathrm{min}$. The detailed procedure of GPC characterizations has been mentioned elsewhere [13].

2.5. Differential Scanning Calorimetry (DSC). Differential scanning calorimetry (DSC, TA Instruments DSC Q1000 series, USA) was used to determine the melting temperature of the polymers. DSC test was carried out in nitrogen environment at a heating rate of $10^{\circ} \mathrm{C} / \mathrm{min}$, where two scans were performed. The thermal properties of polymer depend on the previous history. Therefore, it is a common practice in thermal analysis to heat the samples to remove the previous history and then perform the characterization under the same controlled history. The second scan was used to characterize the polymer samples.

2.6. Dynamic Mechanical Analysis (DMA). DMA (TA instrument, Q800 series, USA) tests of the polymeric samples were performed in single cantilever ( $8 \mathrm{~mm}$ length fixture) mode. The sample specimens with a dimension of $8 \mathrm{~mm}$ in length and $10.1 \mathrm{~mm}$ in width and thickness between 0.65 and
$0.75 \mathrm{~mm}$ were prepared using the fixed size mold in a carver press. The temperature step sweep was varied from 40 to $80^{\circ} \mathrm{C}$ with a step of $10^{\circ} \mathrm{C}$ per each frequency sweep. For frequency sweep, the range of frequency was $0.1-100 \mathrm{~Hz}$ at the strain 15 micron. Temperature and frequency sweep test was run in strain controlled mode. Time-temperature-superposition (TTS) mode was used to analyze the experimental data for getting flow activation energy.

2.7. Mechanical Testing. The mechanical testing of polymeric samples was carried out at room temperature using Instron (model 5567, USA) tensile testing machine at a crosshead speed of $50 \mathrm{~mm} / \mathrm{min}$, where gauge length was kept at $25 \mathrm{~mm}$. The samples were prepared according to ASTM D638 (Type $\mathrm{V}$ ) using a dog-bone mold in carver press.

\section{Results and Discussion}

3.1. Effect of Polymerization Conditions on $M W$ and MWD of Ethylene/1-Butene Copolymers. The variation of different polymerization conditions on MW and MWD of ethylene/1butene copolymers has been shown in Table 1 . The effect of hydrogen pressure on MW and MWD has been investigated at 1,2 , and 3 bar pressure. It is seen from the table that, with the increase in hydrogen pressure, there is a decrease in 
copolymer MW and broadening of MWD. This decrease in copolymer MW and broadening of MWD can be explained by considering the role of hydrogen pressure on the polymerization reaction. Actually, the hydrogen present in the polymerization reaction acts as a chain transfer agent for the reaction $[1,14]$. Thus the increase in hydrogen pressure results in the termination of polymerization reaction. This leads to the lowering of copolymer MW and broadening of MWD. Moballegh and Hakim have reported that an increase in hydrogen concentration by $0.26 \mathrm{~mol}$ raised the MWD from 8 to about 22.8 with considerable decrease in copolymer MW [1]. This observation is consistent with our results mentioned herein. This decrease in MW and broadening of MWD with respect to hydrogen pressure are observed at both ethylene pressure values ( 5 and 10 bar) and both polymerization temperatures $\left(60\right.$ and $\left.80^{\circ} \mathrm{C}\right)$.

The variation of MW and MWD with respect to ethylene pressure (5 and 10 bar) has been shown in Table 1 . The increase in ethylene pressure increases the copolymer MW and narrows the MWD. MW is found to increase from $119172 \mathrm{~g} / \mathrm{mol}$ to $123435 \mathrm{~g} / \mathrm{mol}$ (sample sets numbers 5 and 17) when ethylene pressure was increased from 5 to 10 bar while other polymerization conditions were kept the same. This change represents an increase of $3.45 \%$ in $\mathrm{MW}$ and reduction in MWD from 3.46 to 3.27 (5.8\% reduction in MWD). This decrement/increment level of percentage for MW and MWD varies with the sample sets. The ethylene present in the polymerization reaction acts as an activator for the catalyst in the reaction [14]. At high ethylene pressure, the role of ethylene as an activator is increased which results in copolymer with high MW and narrow MWD.

Table 1 shows that MW and MWD of copolymer are also affected by polymerization temperature. An increase in polymerization temperature from 60 to $80^{\circ} \mathrm{C}$ results in the lowering of MW and broadening of MWD, which can be attributed to the strong chain transfer reaction during copolymerization. The effect of comonomer concentration (1butene) on MW and MWD has been reported at two different concentrations in the table. It is seen that the increase in comonomer concentration from 71.3 to $142.6 \mathrm{mmol} / \mathrm{L}$ results in a decrease in MW and broadening of MWD due to the chain transfer reaction mechanism [1]. In fact, the increase in comonomer concentration leads to a decrease in the catalyst activity as reported in the literature [15-17].

3.2. Dynamic Mechanical Analysis (DMA). The effects of frequency and temperature on dynamic mechanical properties like storage modulus, $G^{\prime}$, and $\tan \delta$ (damping factor) have been presented for the copolymer sample 2 (polymerized at ethylene pressure $=5$ bar; hydrogen pressure $=2$ bar; polymerization temperature $=60^{\circ} \mathrm{C}$; and polymerization time $=30 \mathrm{~min}$ ). The frequency has been varied from 0.1 to $100 \mathrm{~Hz}$ where the temperatures are $50^{\circ} \mathrm{C}, 60^{\circ} \mathrm{C}, 70^{\circ} \mathrm{C}$, and $80^{\circ} \mathrm{C}$. The variation of $G^{\prime}$ versus frequency measured at different temperatures is presented in Figure 1. There is almost a linear increase in $G^{\prime}$ with the increase in frequency. On the contrary, $G^{\prime}$ is found to decrease with the increase in temperature steps. This is due to softening of copolymer at

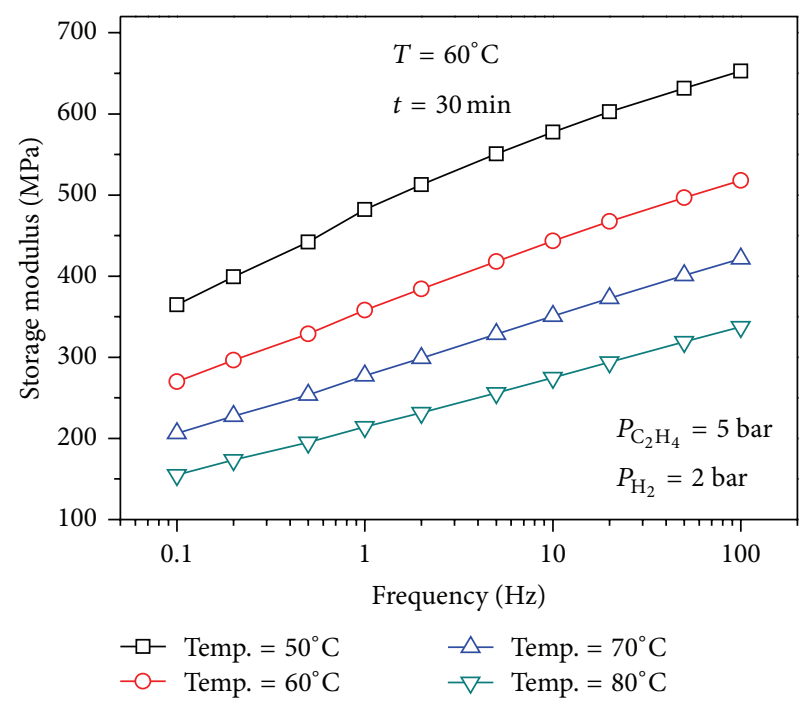

FIGURE 1: Dynamic temperature step/frequency sweep for copolymer sample 2 synthesized at $P_{\mathrm{C}_{2} \mathrm{H}_{4}}=5 \mathrm{bar}, P_{\mathrm{H}_{2}}=2 \mathrm{bar}, T_{\text {polymerization }}$ temperature $=60^{\circ} \mathrm{C}$, and $t_{\text {polymerization }}$ time $=30 \mathrm{~min}$.

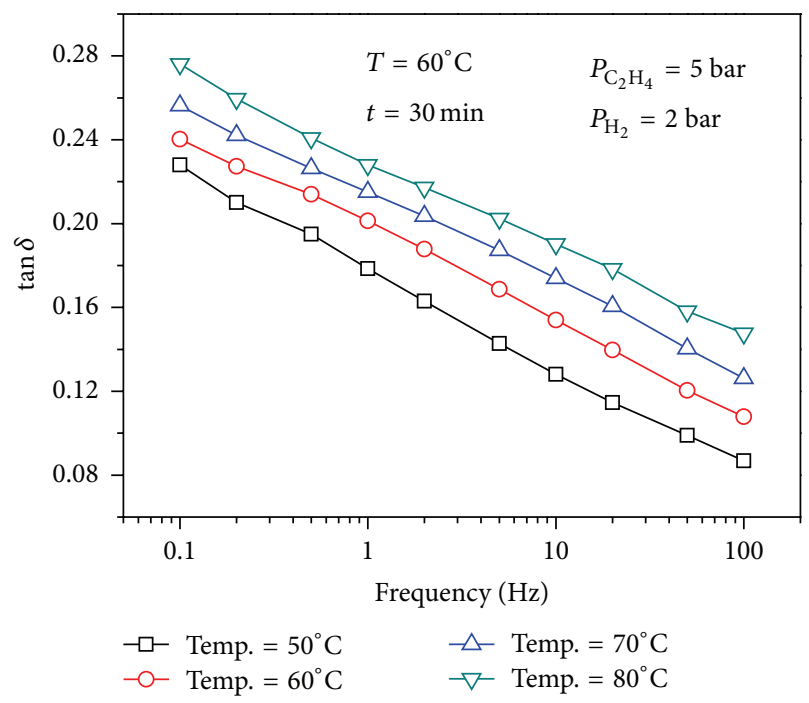

FIgURE 2: Dynamic temperature step/frequency sweep $(\tan \delta)$ for copolymer sample 2 synthesized at $P_{\mathrm{C}_{2} \mathrm{H}_{4}}=5 \mathrm{bar}, P_{\mathrm{H}_{2}}=2 \mathrm{bar}$, $T_{\text {polymerization }}$ temperature $=60^{\circ} \mathrm{C}$, and $t_{\text {polymerization }}$ time $=30 \mathrm{~min}$.

high temperature, which leads to lowering of storage energy and thus $G^{\prime}$. Figure 2 shows the effect of frequency on $\tan \delta$ at different temperatures. It is observed that $\tan \delta$ value decreases with the increase in frequency but increases with the increase in temperature. This increment in $\tan \delta$ with the increase in temperature is due to loss in storage energy at higher temperature.

The effect of frequency on $G^{\prime}$ at different hydrogen pressure, namely, 1, 2, and 3 bar, for the samples 1, 2, and 3 has been presented in Figure 3. The copolymerization conditions for these samples are the same (ethylene pressure $=5 \mathrm{bar}$; polymerization temperature $=60^{\circ} \mathrm{C}$; and 


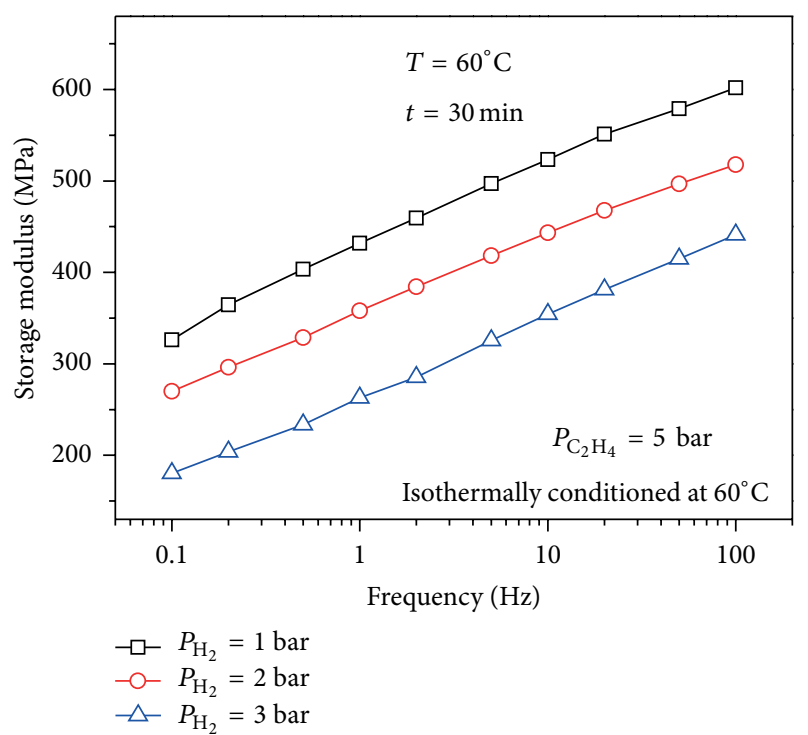

FIGURE 3: Storage modulus of copolymers synthesized at $P_{\mathrm{C}_{2} \mathrm{H}_{4}}=5$ bar, $T_{\text {polymerization }}=60^{\circ} \mathrm{C}$, and $t_{\text {polymerization }}=30 \mathrm{~min}$ (samples 1,2 , and $3)$.

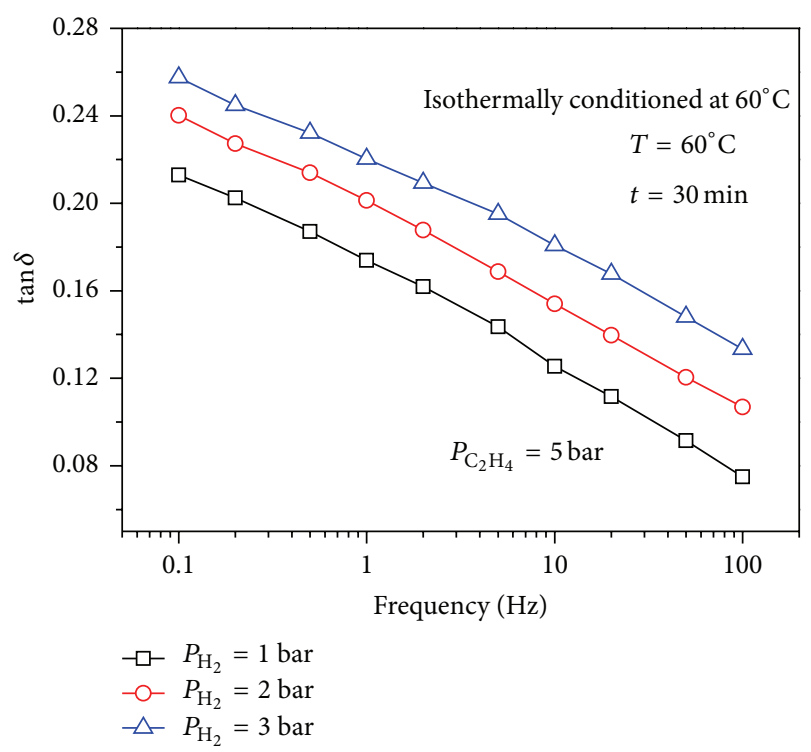

Figure 4: $\tan \delta$ of copolymers synthesized at $P_{\mathrm{C}_{2} \mathrm{H}_{4}}=5$ bar, $T_{\text {polymerization }}=60^{\circ} \mathrm{C}$, and $t_{\text {polymerization }}=30 \mathrm{~min}$ (samples 1,2 , and 3 ).

polymerization time $=30 \mathrm{~min}$ ). The increase in hydrogen pressure results in reduction of $G^{\prime}$ as is observed from the figure. Actually, the hydrogen present in the reaction system acts as an efficient chain transfer agent for the reaction as has been mentioned earlier. This leads to copolymer with short chain length having lower MW. In fact, the degree of chain entanglements in polymer decreases with the decrease in MW and consequently results in lower value of $G^{\prime}$. Figure 4 shows $\tan \delta$ against frequency plots at different hydrogen pressure for the same set of samples. It is observed from

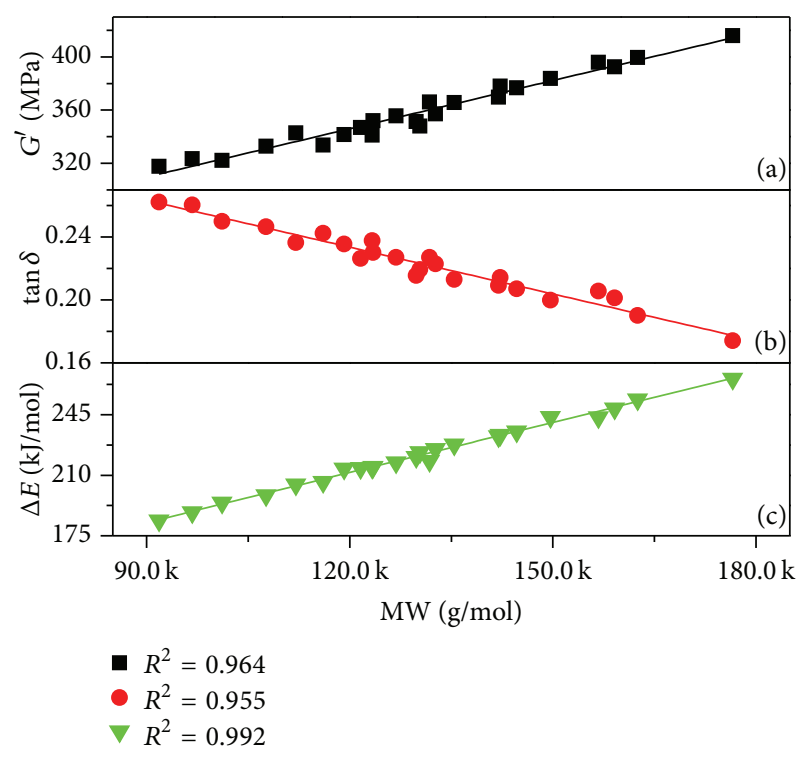

FIGURE 5: Correlation plots of MW with $G^{\prime}$ (a), $\tan \delta(\mathrm{b})$, and $\Delta E$ (c).

this figure that the increase in hydrogen pressure leads to increase in $\tan \delta$ value at any particular frequency. In fact, the polymer with lower MW (short chain length) will have higher molecular movement compared to high MW polymer of the same category. This is because the polymer with low MW will have low surface area per unit chain length, which results in low physical attraction between the molecules [18]. Thus the increase in molecular movement in copolymer results in higher value of $\tan \delta$, as $\tan \delta$ is a measure of structural transformation (molecular movement) in polymer. The higher the value of $\tan \delta$ is, the higher the structural transformation in polymer will be. This is the reason for low molecular weight polymer (short chain length polymer) having higher value of $\tan \delta$ compared to long chain length polymer. The effects of ethylene pressure and polymerization temperature on both $G^{\prime}$ and $\tan \delta$ of the polymer are almost similar (not shown in picture). The differences exist only in their magnitude. The variations of $G^{\prime}$ and $\tan \delta$ with respect to frequency/temperature sweep, ethylene and hydrogen pressure, and polymerization temperature have been investigated for all samples but are not reported in this paper because the trend in variations is similar for all the samples. The correlation of $G^{\prime}$ and $\tan \delta$ with MW of polymers has been established and the linear fit of the plots is shown in Figures 5(a) and 5(b). The increase in MW leads to increase in $G^{\prime}$ which results in a reduction in $\tan \delta$ values. The coefficients of correlation $\left(R^{2}\right)$ of the linear fit for $G^{\prime}$ and $\tan \delta$ are 0.964 and 0.955 , respectively. Both $R^{2}$ are almost close to unity, indicating good linear correlation of $G^{\prime}$ and $\tan \delta$ with MW.

The time temperature superposition (TTS) technique has been applied to get the activation energy $(\Delta E)$ for all the samples in solid state extracted from the DMA data. The results of activation energy have been presented in Table 2. The activation energy for all samples falls in the range of $183.9-265.8 \mathrm{~kJ} / \mathrm{mol}$. It is seen that the sample with higher MW exhibits higher $\Delta E$. The data of $\Delta E$ are plotted against 
TABLE 2: Activation energy for copolymer samples (polymerization time: $30 \mathrm{~min}$ ).

\begin{tabular}{|c|c|c|c|c|c|c|}
\hline s/number & $P_{\mathrm{C}_{2} \mathrm{H}_{4}}$ (bar) & 1-Butene fraction (mmol) & $\begin{array}{c}\text { Poly. } \\
\left.\text { temp. ( }{ }^{\circ} \mathrm{C}\right)\end{array}$ & $P_{\mathrm{H}_{2}}$ (bar) & Activation energy $(\mathrm{kJ} / \mathrm{mol})$ & $\mathrm{MW}(\mathrm{g} / \mathrm{mol})$ \\
\hline 1 & 5 & 71.3 & 60 & 1 & 253.9 & 162534 \\
\hline 2 & 5 & 71.3 & 60 & 2 & 243.7 & 149653 \\
\hline 3 & 5 & 71.3 & 60 & 3 & 214.4 & 123342 \\
\hline 4 & 5 & 71.3 & 80 & 1 & 225.1 & 132669 \\
\hline 5 & 5 & 71.3 & 80 & 2 & 213.9 & 119172 \\
\hline 6 & 5 & 71.3 & 80 & 3 & 198.4 & 107637 \\
\hline 7 & 5 & 142.6 & 60 & 1 & 235.4 & 144653 \\
\hline 8 & 5 & 142.6 & 60 & 2 & 223.4 & 130350 \\
\hline 9 & 5 & 142.6 & 60 & 3 & 214.1 & 121622 \\
\hline 10 & 5 & 142.6 & 80 & 1 & 220.5 & 129823 \\
\hline 11 & 5 & 142.6 & 80 & 2 & 194.1 & 101153 \\
\hline 12 & 5 & 142.6 & 80 & 3 & 183.9 & 91842 \\
\hline 13 & 10 & 71.3 & 60 & 1 & 265.8 & 176583 \\
\hline 14 & 10 & 71.3 & 60 & 2 & 248.6 & 159136 \\
\hline 15 & 10 & 71.3 & 60 & 3 & 218.3 & 131776 \\
\hline 16 & 10 & 71.3 & 80 & 1 & 232.6 & 141970 \\
\hline 17 & 10 & 71.3 & 80 & 2 & 214.8 & 123435 \\
\hline 18 & 10 & 71.3 & 80 & 3 & 204.7 & 112062 \\
\hline 19 & 10 & 142.6 & 60 & 1 & 243.6 & 156754 \\
\hline 20 & 10 & 142.6 & 60 & 2 & 233.4 & 142238 \\
\hline 21 & 10 & 142.6 & 60 & 3 & 217.4 & 126835 \\
\hline 22 & 10 & 142.6 & 80 & 1 & 227.8 & 135454 \\
\hline 23 & 10 & 142.6 & 80 & 2 & 206.1 & 116068 \\
\hline 24 & 10 & 142.6 & 80 & 3 & 188.6 & 96752 \\
\hline
\end{tabular}

MW and are shown in Figure 5(c), which reveals a linear relationship of $\Delta E$ with MW. The value of $R^{2}(0.992)$ is very close to unity, indicating that $\mathrm{MW}$ is the single parameter which influences $\Delta E$.

3.3. Differential Scanning Calorimeter (DSC). The thermal properties of copolymer, that is, the crystallization temperature $(\mathrm{Tc})$, melting temperature $(\mathrm{Tm})$, and \% crystallinity (\%Xc), have been extracted from DSC and are shown in Table 3. The results show that Tc of copolymer remains in the range of $114.69-117.89^{\circ} \mathrm{C}$. This implies that the effects of polymerization parameters, covered in this study, on Tc are marginal.

The effect of hydrogen pressure can be correlated with copolymer melting point as can be seen from the table. It is observed that Tm decreases with the increase in hydrogen pressure. However, this decrement in melting point is less significant. This decrement in melting point with the increase in hydrogen pressure can be explained by considering the role of hydrogen as chain transfer agent during reaction which already has been mentioned earlier. This role of hydrogen leads to lower MW copolymer that means lower surface area of copolymer. Thus, there will be less physical attraction between low MW polymers chains for which less energy is required to overcome this force of attraction [18]. This is why, with the increase in hydrogen pressure, the melting point of the copolymer decreases. The ethylene pressure has less impact on melting point of copolymer. It is seen from the table that $\mathrm{Tm}$ at 5 bar of ethylene pressure is in the range of $123.99-130.12^{\circ} \mathrm{C}$, whereas at 10 bar of ethylene pressure is in the range of $125.91-133.14^{\circ} \mathrm{C}$. Thus a little bit positive shift of temperature is observed when ethylene pressure is increased. This positive shift may be due to narrow MWD of polymers synthesized at high ethylene pressure. Similarly, the effect of polymerization temperature on copolymer melting point is less significant as is observed from the table.

The effect of polymerization parameters on copolymer crystallinity has been reported in the same table as is observed in the last column. It is revealed from this table that the copolymer crystallinity increases with the increase in hydrogen pressure. As the coordination polymerization method is processed through successive polymerization and crystallization [19], the increase in hydrogen pressure blocks the active sites of polymerization resulting in decrease in polymerization rate; as a consequence, the crystallization rate is increased. This is why the increase in hydrogen pressure leads to increase in crystallinity [20]. An almost similar observation has been made for ethylene pressure; that is, the increment in copolymer crystallinity is less significant with the increase in ethylene pressure from 5 to 10 bar 
TABLE 3: DSC analysis for copolymer samples (polymerization time $30 \mathrm{~min}$ ).

\begin{tabular}{|c|c|c|c|c|c|c|c|}
\hline s/number & $P_{\mathrm{C}_{2} \mathrm{H}_{4}}$ (bar) & 1-Butene fraction $(\mathrm{mmol})$ & $\begin{array}{c}\text { Poly. } \\
\text { temp. }\left({ }^{\circ} \mathrm{C}\right)\end{array}$ & $P_{\mathrm{H}_{2}}$ (bar) & $T_{c}\left({ }^{\circ} \mathrm{C}\right)$ & $T_{m}\left({ }^{\circ} \mathrm{C}\right)$ & $\%$ cryst. (cooling) \\
\hline 1 & 5 & 71.3 & 60 & 1 & 117.89 & 130.12 & 46.32 \\
\hline 2 & 5 & 71.3 & 60 & 2 & 116.86 & 128.76 & 49.54 \\
\hline 3 & 5 & 71.3 & 60 & 3 & 115.52 & 125.92 & 53.86 \\
\hline 4 & 5 & 71.3 & 80 & 1 & 116.91 & 128.36 & 48.13 \\
\hline 5 & 5 & 71.3 & 80 & 2 & 115.42 & 127.37 & 52.03 \\
\hline 6 & 5 & 71.3 & 80 & 3 & 114.37 & 124.73 & 56.57 \\
\hline 7 & 5 & 142.6 & 60 & 1 & 116.83 & 129.54 & 48.60 \\
\hline 8 & 5 & 142.6 & 60 & 2 & 115.52 & 127.31 & 52.44 \\
\hline 9 & 5 & 142.6 & 60 & 3 & 114.63 & 124.82 & 55.58 \\
\hline 10 & 5 & 142.6 & 80 & 1 & 115.34 & 127.43 & 50.83 \\
\hline 11 & 5 & 142.6 & 80 & 2 & 114.45 & 125.86 & 53.61 \\
\hline 12 & 5 & 142.6 & 80 & 3 & 113.92 & 123.99 & 56.63 \\
\hline 13 & 10 & 71.3 & 60 & 1 & 119.55 & 133.14 & 51.75 \\
\hline 14 & 10 & 71.3 & 60 & 2 & 118.56 & 131.81 & 54.86 \\
\hline 15 & 10 & 71.3 & 60 & 3 & 117.94 & 129.77 & 57.08 \\
\hline 16 & 10 & 71.3 & 80 & 1 & 118.34 & 131.99 & 53.19 \\
\hline 17 & 10 & 71.3 & 80 & 2 & 116.57 & 129.68 & 56.83 \\
\hline 18 & 10 & 71.3 & 80 & 3 & 115.29 & 128.40 & 59.12 \\
\hline 19 & 10 & 142.6 & 60 & 1 & 118.67 & 130.83 & 53.21 \\
\hline 20 & 10 & 142.6 & 60 & 2 & 116.79 & 128.90 & 56.50 \\
\hline 21 & 10 & 142.6 & 60 & 3 & 115.80 & 126.09 & 61.63 \\
\hline 22 & 10 & 142.6 & 80 & 1 & 116.77 & 128.93 & 55.66 \\
\hline 23 & 10 & 142.6 & 80 & 2 & 115.12 & 127.09 & 59.10 \\
\hline 24 & 10 & 142.6 & 80 & 3 & 114.69 & 125.91 & 63.62 \\
\hline
\end{tabular}

as is observed from the table. However, the increment in crystallinity varies according to the condition of polymerization. Shin et al. have also reported similar results that the increase in ethylene pressure leads to marginal increase in crystallinity [21]. The variation of copolymer crystallinity with the increase in polymerization temperature is also marginal as is observed from the table.

The influence of MW on polymer Tm, Tc, and \%Xc has been investigated and the related plots are presented in Figures 6(a)-6(c). It is observed from the figure that, for all the three cases, though the data points are scattered randomly, there are the overall improvement in Tm (Figure 6(a)) and Tc (Figure 6(b)) and reduction in \%Xc (Figure 6(c)) with the increase in MW. The $R^{2}$ of the linear fits for Tm, Tc, and $\% \mathrm{Xc}$ are $0.39,0.49$, and 0.65 , respectively, far away from the unity. Thus it can be said that the polymers Tm, Tc, and \%Xc are not only influenced by MW but also dependent on other parameters.

3.4. Mechanical Properties. The results of mechanical properties of copolymers like tensile modulus (TM), tensile strength (TS), and \% strain at break (SB) are affected by the polymerization parameters. Table 4 shows the mechanical testing results of copolymer for all the samples. It is seen that the increase in hydrogen pressure results in the increase

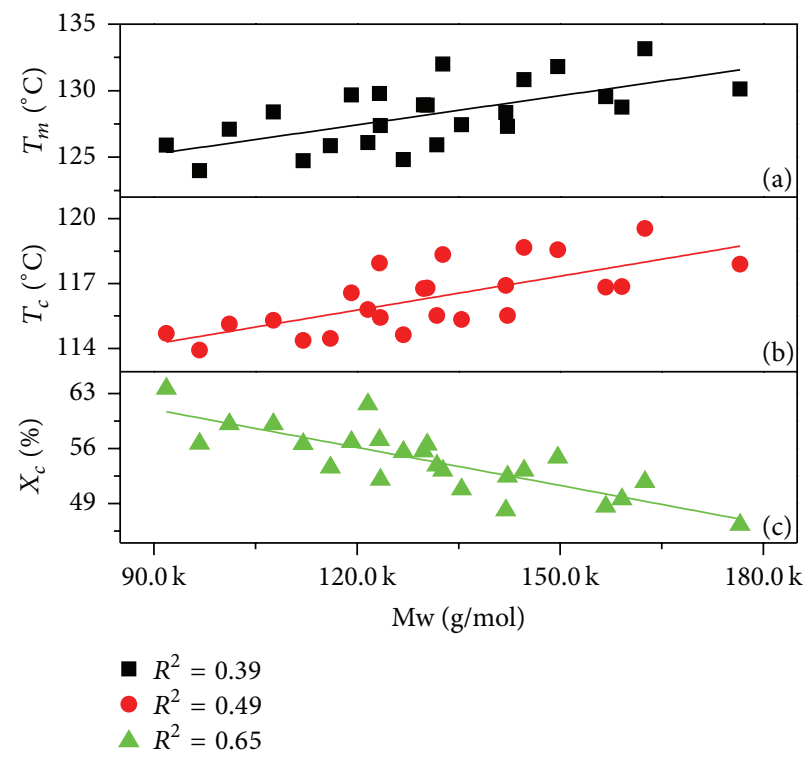

FIgure 6: Correlation plots of MW with Tm (a), Tc (b), and \%Xc (c).

in tensile modulus. This type of result is obtained when polymerization is carried out at both 5 and 10 bar ethylene 
TABLE 4: Mechanical properties of ethylene/1-butene copolymer (polymerization time: $30 \mathrm{~min}$ ).

\begin{tabular}{|c|c|c|c|c|c|c|c|c|c|}
\hline s/number & $\begin{array}{c}P_{\mathrm{C}_{2} \mathrm{H}_{4}} \\
\text { (bar) }\end{array}$ & $\begin{array}{c}\text { 1-Butene fraction } \\
(\mathrm{mmol} / \mathrm{L})\end{array}$ & $\begin{array}{c}\text { Poly. } \\
\text { temp. }\left({ }^{\circ} \mathrm{C}\right)\end{array}$ & $P_{\mathrm{H}_{2}}$ (bar) & $\begin{array}{l}\text { Modulus } \\
(\mathrm{MPa})\end{array}$ & $\begin{array}{c}\text { Yield stress } \\
(\mathrm{MPa})\end{array}$ & $\begin{array}{c}\text { Yield strain } \\
(\%)\end{array}$ & $\begin{array}{c}\text { Strain at } \\
\text { break }(\%)\end{array}$ & $\mathrm{TS}(\mathrm{MPa})$ \\
\hline 1 & 5 & 71.3 & 60 & 1 & $419 \pm 18$ & $13.63 \pm 1.13$ & 3 & $669 \pm 23$ & $48.65 \pm 4.48$ \\
\hline 2 & 5 & 71.3 & 60 & 2 & $514 \pm 21$ & $15.12 \pm 1.25$ & 3 & $756 \pm 27$ & $45.55 \pm 3.56$ \\
\hline 3 & 5 & 71.3 & 60 & 3 & $591 \pm 28$ & $17.08 \pm 1.32$ & 3 & $817 \pm 35$ & $41.42 \pm 3.24$ \\
\hline 4 & 5 & 71.3 & 80 & 1 & $445 \pm 15$ & $14.12 \pm 1.21$ & 4 & $686 \pm 32$ & $47.91 \pm 4.25$ \\
\hline 5 & 5 & 71.3 & 80 & 2 & $544 \pm 20$ & $15.22 \pm 1.27$ & 3 & $790 \pm 28$ & $43.89 \pm 3.96$ \\
\hline 6 & 5 & 71.3 & 80 & 3 & $615 \pm 31$ & $17.29 \pm 1.31$ & 3 & $845 \pm 39$ & $39.89 \pm 3.47$ \\
\hline 7 & 5 & 142.6 & 60 & 1 & $437 \pm 17$ & $14.05 \pm 1.12$ & 4 & $678 \pm 25$ & $47.32 \pm 4.14$ \\
\hline 8 & 5 & 142.6 & 60 & 2 & $536 \pm 22$ & $15.58 \pm 1.18$ & 4 & $771 \pm 29$ & $43.45 \pm 3.82$ \\
\hline 9 & 5 & 142.6 & 60 & 3 & $620 \pm 29$ & $17.21 \pm 1.24$ & 4 & $823 \pm 33$ & $40.24 \pm 3.32$ \\
\hline 10 & 5 & 142.6 & 80 & 1 & $499 \pm 20$ & $14.18 \pm 1.14$ & 4 & $698 \pm 34$ & $46.55 \pm 4.06$ \\
\hline 11 & 5 & 142.6 & 80 & 2 & $565 \pm 27$ & $15.75 \pm 1.19$ & 4 & $785 \pm 37$ & $42.61 \pm 3.75$ \\
\hline 12 & 5 & 142.6 & 80 & 3 & $648 \pm 33$ & $17.51 \pm 1.25$ & 3 & $842 \pm 42$ & $38.64 \pm 3.26$ \\
\hline 13 & 10 & 71.3 & 60 & 1 & $458 \pm 16$ & $13.81 \pm 1.11$ & 3 & $687 \pm 26$ & $51.94 \pm 4.57$ \\
\hline 14 & 10 & 71.3 & 60 & 2 & $535 \pm 23$ & $15.34 \pm 1.22$ & 3 & $784 \pm 35$ & $46.65 \pm 4.12$ \\
\hline 15 & 10 & 71.3 & 60 & 3 & $634 \pm 29$ & $17.23 \pm 1.29$ & 3 & $843 \pm 39$ & $42.67 \pm 3.35$ \\
\hline 16 & 10 & 71.3 & 80 & 1 & $472 \pm 19$ & $14.62 \pm 1.13$ & 3 & $702 \pm 33$ & $49.43 \pm 4.36$ \\
\hline 17 & 10 & 71.3 & 80 & 2 & $575 \pm 25$ & $15.58 \pm 1.20$ & 3 & $798 \pm 37$ & $44.68 \pm 3.94$ \\
\hline 18 & 10 & 71.3 & 80 & 3 & $682 \pm 34$ & $17.47 \pm 1.26$ & 3 & $856 \pm 41$ & $41.53 \pm 3.28$ \\
\hline 19 & 10 & 142.6 & 60 & 1 & $481 \pm 23$ & $14.27 \pm 1.15$ & 3 & $698 \pm 29$ & $49.84 \pm 4.19$ \\
\hline 20 & 10 & 142.6 & 60 & 2 & $554 \pm 27$ & $15.74 \pm 1.23$ & 4 & $812 \pm 36$ & $44.66 \pm 3.86$ \\
\hline 21 & 10 & 142.6 & 60 & 3 & $665 \pm 34$ & $17.67 \pm 1.31$ & 3 & $876 \pm 43$ & $41.83 \pm 3.42$ \\
\hline 22 & 10 & 142.6 & 80 & 1 & $512 \pm 29$ & $14.63 \pm 1.17$ & 3 & $718 \pm 25$ & $47.45 \pm 4.14$ \\
\hline 23 & 10 & 142.6 & 80 & 2 & $589 \pm 36$ & $16.42 \pm 1.28$ & 3 & $828 \pm 37$ & $43.36 \pm 3.68$ \\
\hline 24 & 10 & 142.6 & 80 & 3 & $712 \pm 39$ & $18.19 \pm 1.33$ & 3 & $896 \pm 42$ & $40.35 \pm 3.16$ \\
\hline
\end{tabular}

pressure. When polymerization is performed at 5 bar ethylene pressure, the modulus is in the range of $419 \pm 18-499 \pm$ $20 \mathrm{MPa}$ at 1 bar hydrogen pressure, $514 \pm 21-565 \pm 27 \mathrm{MPa}$ at 2 bar hydrogen pressure, and $591 \pm 28-648 \pm 33 \mathrm{MPa}$ at 3 bar hydrogen pressure. Almost similar results are obtained when polymerization condition is maintained at 10 bar ethylene pressure, where the tensile modulus is in the range of $458 \pm$ $16-512 \pm 29 \mathrm{MPa}$ at 1 bar hydrogen pressure, $535 \pm 23-589$ $\pm 36 \mathrm{MPa}$ at 2 bar hydrogen pressure, and $634 \pm 29-712 \pm$ $39 \mathrm{MPa}$ at 3 bar hydrogen pressure. Thus a careful look at the results reveals that the tensile modulus value at 10 bar ethylene pressure is higher compared to tensile modulus value at 5 bar ethylene pressure. Actually, the tensile modulus is related to copolymer crystallinity as shown in Table 3. It is seen that the copolymer with higher crystallinity is having higher modulus.

The results of \% strain at break reported in the table show an increment in \% strain at break with the increase in hydrogen pressure. The $\%$ strain at break at 1 bar hydrogen pressure is in the range of $669 \pm 23-698 \pm 34$, at 2 bar hydrogen pressure is in the range $756 \pm 27-785 \pm 37$, and at 3 bar hydrogen pressure is in the range of $817 \pm 35-842 \pm 42$, when polymerization is conditioned at 5 bar ethylene pressure. Similarly, when polymerization is conditioned at 10 bar ethylene pressure, the range of $\%$ strain at break at 1 bar hydrogen pressure is $687 \pm 26-718 \pm 25$, at 2 bar hydrogen pressure is $784 \pm 35-828 \pm 37$, and at 3 bar hydrogen pressure is $843 \pm 39-896 \pm 42$. Thus the $\%$ strain at break is somewhat high for the copolymer synthesis at higher ethylene pressure. It is observed from the table that the yield strain remains in between 3 and $4 \%$. A careful look at Tables 4 and 3 reveals that the copolymer with low MW is having higher \% strain at break.

Some variation of yield stress (YS) with the increase in hydrogen pressure is observed from the results shown in the same table. When polymerization is conditioned at 5 bar ethylene pressure, the yield stress ranges at 1 bar, 2 bar, and 3 bar hydrogen pressure are $13.63 \pm 1.13-14.18 \pm 1.14 \mathrm{MPa}$, $15.12 \pm 1.25-15.75 \pm 1.19 \mathrm{MPa}$, and $17.08 \pm 1.32-17.51 \pm 1.25 \mathrm{MPa}$, respectively. This shows an increment in yield stress with the increase in hydrogen pressure. A similar type of increment is observed when polymerization is conditioned at $10 \mathrm{bar}$ ethylene pressure; that is, the yield stress ranges are 13.81 $\pm 1.11-14.63 \pm 1.17 \mathrm{MPa}, 15.34 \pm 1.22-16.42 \pm 1.28 \mathrm{MPa}$, and $17.23 \pm 1.29-18.19 \pm 1.33 \mathrm{MPa}$ at 1 bar, 2 bar, and 3 bar hydrogen pressure, respectively. So, from these results it comes true that the effect of ethylene pressure on yield stress is less significant compared to the effect of hydrogen pressure. It is also evident from the table that the polymerization temperature has low impact on yield stress. The tensile strength of copolymer 


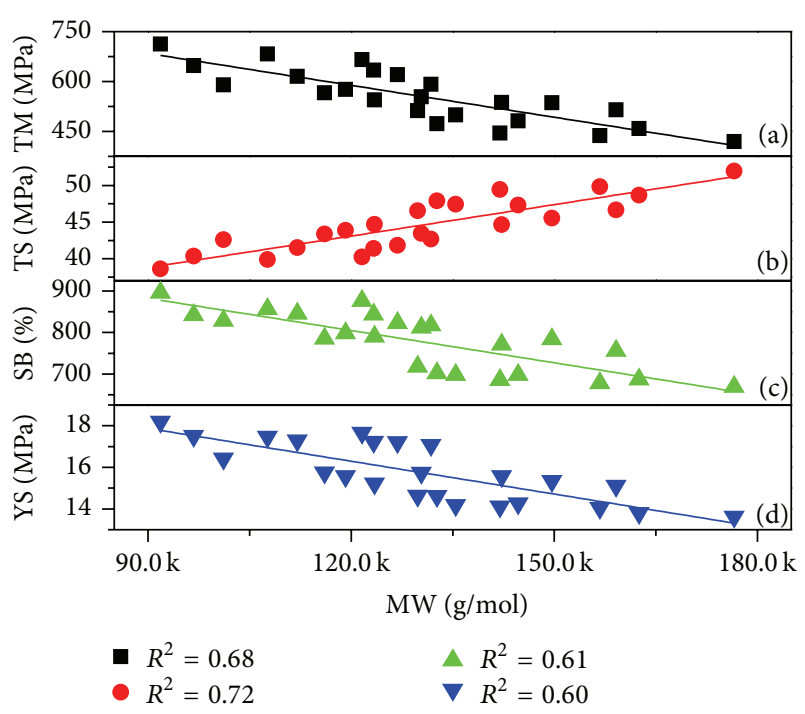

FIGURE 7: Correlation plots of MW with TM (a), TS (b), SB (c), and YS (d).

improves with the increase in ethylene pressure but decreases with the increase in hydrogen pressure and polymerization temperature as is observed from the table.

The data of tensile modulus (TM), tensile strength (TS), $\%$ strain at break (SB), and yield stress (YS) are plotted against MW and are shown in Figures $7(\mathrm{a})-7(\mathrm{~d})$, respectively. An effort to make linear fits of randomly scattered data points has been made for all four cases. There is overall decrement in TM, SB, and YS with the increase in MW. The improvement happened only for TS. The $R^{2}$ of linear fits for TM (Figure 7(a)), TS (Figure 7(b)), SB (Figure 7(c)), and YS (Figure 7(d)) are $0.68,0.72,0.61$, and 0.60 , respectively. These $R^{2}$ values are not close to unity, indicating that these mechanical properties not only depend on MW but are also influenced by other parameters. Actually, rather than MW, these mechanical properties also depend on several parameters like polymer crystallinity, backbone chain length, branch (side) chain length, chain entanglement, branch chain content, and so forth $[4,12,22-25]$. Thus the establishment of perfect correlation of MW with these mechanical properties is really difficult.

\section{Conclusions}

The increase in both hydrogen pressure and polymerization temperature leads to decrease in copolymer MW and broadening of MWD. But the ethylene pressure plays the opposite role. DMA results show an increase in storage modulus but decrease in $\tan \delta$ value with the increase in frequency. On the contrary, the storage modulus value decreases but $\tan \delta$ value increases with the increase in temperature. An increase in hydrogen pressure results in reduction of copolymer storage modulus and melting point but increase in crystallinity, tensile modulus, \% strain at break, and yield stress. It is seen that the copolymer with higher MW exhibits higher activation energy. It is revealed that the copolymer with higher crystallinity is having higher tensile modulus. However, the impacts of other polymerization parameters like ethylene pressure and polymerization temperature on copolymer modulus, tensile strength, crystallinity, Tc, yield stress, \% strain at break, and yield strain are marginal. The major role of hydrogen in controlling the copolymer properties is due to its acting as an efficient chain transfer agent during polymerization reaction. The correlation of MW with the thermal and mechanical properties of the polymer is given in this paper. The MW is the key parameter that influences $\Delta E$, whereas the other dynamic mechanical $\left(G^{\prime}\right.$ and $\tan \delta$ ), thermal (Tm, Tc, and \%Xc), and mechanical (TM, TS, SB, and YS) properties not only depend on MW but are also influenced by different parameters as mentioned earlier. However, almost good linear correlation of MW with $G^{\prime}$ and $\tan \delta$ is observed.

\section{Conflict of Interests}

The authors declare that there is no conflict of interests regarding the publication of this paper.

\section{Acknowledgments}

The authors acknowledge the financial support provided by King Abdul Aziz City for Science and Technology for this research project under research Grant no. AT-27-67. The authors also acknowledge the facilities and support provided by KFUPM.

\section{References}

[1] L. Moballegh and S. Hakim, "Molecularweight bimodality of ethylene/1-butene in a two-step polymerization process: effects of polymerization conditions," Iranian Polymer Journal, vol. 20, no. 6, pp. 513-521, 2011.

[2] H. S. Mobarakeh, M. F. Monfared, and M. Vakili, "Gas phase copolymerization of ethylene and 1-butene with prepolymerized $\mathrm{MgCl}_{2}$ supported Ziegler-Natta catalyst: effect of $\mathrm{Al} / \mathrm{Ti}$ ratio," Iranian Polymer Journal, vol. 15, no. 7, pp. 569-575, 2006.

[3] W. Kaminsky, "New polymers by metallocene catalysis," Macromolecular Chemistry and Physics, vol. 197, no. 12, pp. 3907-3945, 1996.

[4] D.-P. Quan, X.-G. Fan, and H.-H. Wang, "Structure and mechanical properties of ethylene-butene copolymers," Chemical Research in Chinese Universities, vol. 18, no. 1, pp. 52-55, 2002.

[5] Y. V. Kissin, R. I. Mink, T. E. Nowlin, and A. J. Brandolini, "Kinetics and mechanism of ethylene homopolymerization and copolymerization reactions with heterogeneous Ti-based Ziegler-Natta catalysts," Topics in Catalysis, vol. 7, no. 1-4, pp. 69-88, 1999.

[6] Y. V. Kissin, F. M. Mirabella, and C. C. Meverden, "Multicenter nature of heterogeneous Ziegler-Natta catalysts: TREF confirmation," Journal of Polymer Science A: Polymer Chemistry, vol. 43, no. 19, pp. 4351-4362, 2005.

[7] L. G. Echevskaya, M. A. Matsko, T. B. Mikenas, V. E. Nikitin, and V. A. Zakharov, "Supported titanium-magnesium catalysts with different titanium content: kinetic peculiarities at ethylene homopolymerization and copolymerization and molecular 
weight characteristics of polyethylene," Journal of Applied Polymer Science, vol. 102, no. 6, pp. 5436-5442, 2006.

[8] V. Zakharov, L. Echevskaya, T. Mikenas et al., "Supported ziegler-natta catalysts for ethylene slurry polymerization and control of molecular weight distribution of polyethylene," Chinese Journal of Polymer Science, vol. 26, no. 5, pp. 553-559, 2008.

[9] M. A. Matsko, L. G. Echevskaya, V. A. Zakharov, M. I. Nikolaeva, T. B. Mikenas, and M. P. Vanina, "Study of multi-site nature of supported Ziegler-Natta catalysts in ethylene-hexene1 copolymerization," Macromolecular Symposia, vol. 282, no. 1, pp. 157-166, 2009.

[10] Y. V. Kissin, R. I. Mink, and T. E. Nowlin, "Ethylene polymerization reactions with Ziegler-Natta catalysts. I. Ethylene polymerization kinetics and kinetic mechanism," Journal of Polymer Science A: Polymer Chemistry, vol. 37, pp. 4255-4272, 1999.

[11] P. Kaewarsa, Polymerization of ethylene over the supported Ziegler-Natta and metallocene catalysts on magnesium hydroxide and magnesium hydroxychloride [Thesis in Chemistry Science], Graduate School: Khonkaen University, 2005.

[12] M. A. Parvez, M. Rahaman, M. A. Suleiman, J. B. P. Soares, and I. A. Hussein, "Correlation of polymerization conditions with thermal and mechanical properties of polyethylenes made with Ziegler-Natta catalysts," International Journal of Polymer Science, vol. 2014, Article ID 549031, 10 pages, 2014.

[13] T. Hameed and I. A. Hussein, "Rheological study of the influence of $\mathrm{M} \mathrm{W}$ and comonomer type on the miscibility of m-LLDPE and LDPE blends," Polymer, vol. 43, no. 25, pp. 69116929, 2002.

[14] M. I. Nikolaeva, T. B. Mikenas, M. A. Matsko, L. G. Echevskaya, and V. A. Zakharov, "Ethylene polymerization over supported titanium-magnesium catalysts: effect of polymerization parameters on the molecular weight distribution of polyethylene," Journal of Applied Polymer Science, vol. 122, no. 5, pp. 3092-3101, 2011.

[15] J. C. W. Chien and T. Nozaki, "Ethylene-hexene copolymerization by heterogeneous and homogeneous Ziegler-Natta catalysts and the "comonomer" effect," Journal of Polymer Science A: Polymer Chemistry, vol. 31, no. 1, pp. 227-237, 1993.

[16] P. Kumkaew, L. Wu, P. Praserthdam, and S. E. Wanke, "Rates and product properties of polyethylene produced by copolymerization of 1-hexene and ethylene in the gas phase with (n$\mathrm{BuCp})_{2} \mathrm{ZrCl}_{2}$ on supports with different pore sizes," Polymer, vol. 44, no. 17, pp. 4791-4803, 2003.

[17] K. Heiland and W. Kaminsky, "Comparison of zirconocene and hafnocene catalysts for the polymerization of ethylene and 1butene," Macromolecular Chemistry and Physics, vol. 193, pp. 601-610, 1992.

[18] L. H. Sperling, Introduction to Physical Polymer Science, WileyInterscience, New York, NY, USA, 4th edition, 2006.

[19] B. Wunderlich, "Crystallization during polymerization," Advances in Polymer Science, vol. 5, pp. 568-619, 1968.

[20] A. Muñoz-Escalona and A. Parada, "Factors affecting the nascent structure and morphology of polyethylene obtained by heterogeneous Ziegler-Natta catalysts: 2 . Crystallinity and melting behaviour," Polymer, vol. 20, no. 7, pp. 859-866, 1979.

[21] Y.-J. Shin, H.-X. Zhang, K.-B. Yoon, and D.-H. Lee, "Preparation of ultra high molecular weight polyethylene with $\mathrm{MgCl}_{2} / \mathrm{TiCl}_{4}$ catalysts: effect of temperature and pressure," Macromolecular Research, vol. 18, no. 10, pp. 951-955, 2010.

[22] M. F. Talbott, G. S. Springer, and L. A. Berglund, "The effects of crystallinity on the mechanical properties of PEEK polymer and graphite fiber reinforced PEEK,' Journal of Composite Materials, vol. 21, no. 11, pp. 1056-1081, 1987.

[23] J. A. Brydson, Plastics Materials, British Library Cataloguing in Publication Data, 7th edition, 1999.

[24] G. Wang, B. Guo, and R. Li, "Synthesis, characterization, and properties of long-chain branched poly(butylene succinate)," Journal of Applied Polymer Science, vol. 124, no. 2, pp. 1271-1280, 2012.

[25] U. W. Gedde, Polymer Physics, Library of Congress Catalogingin-Publication Data, Kluwer Academic Publishers, 1st edition, 1995. 

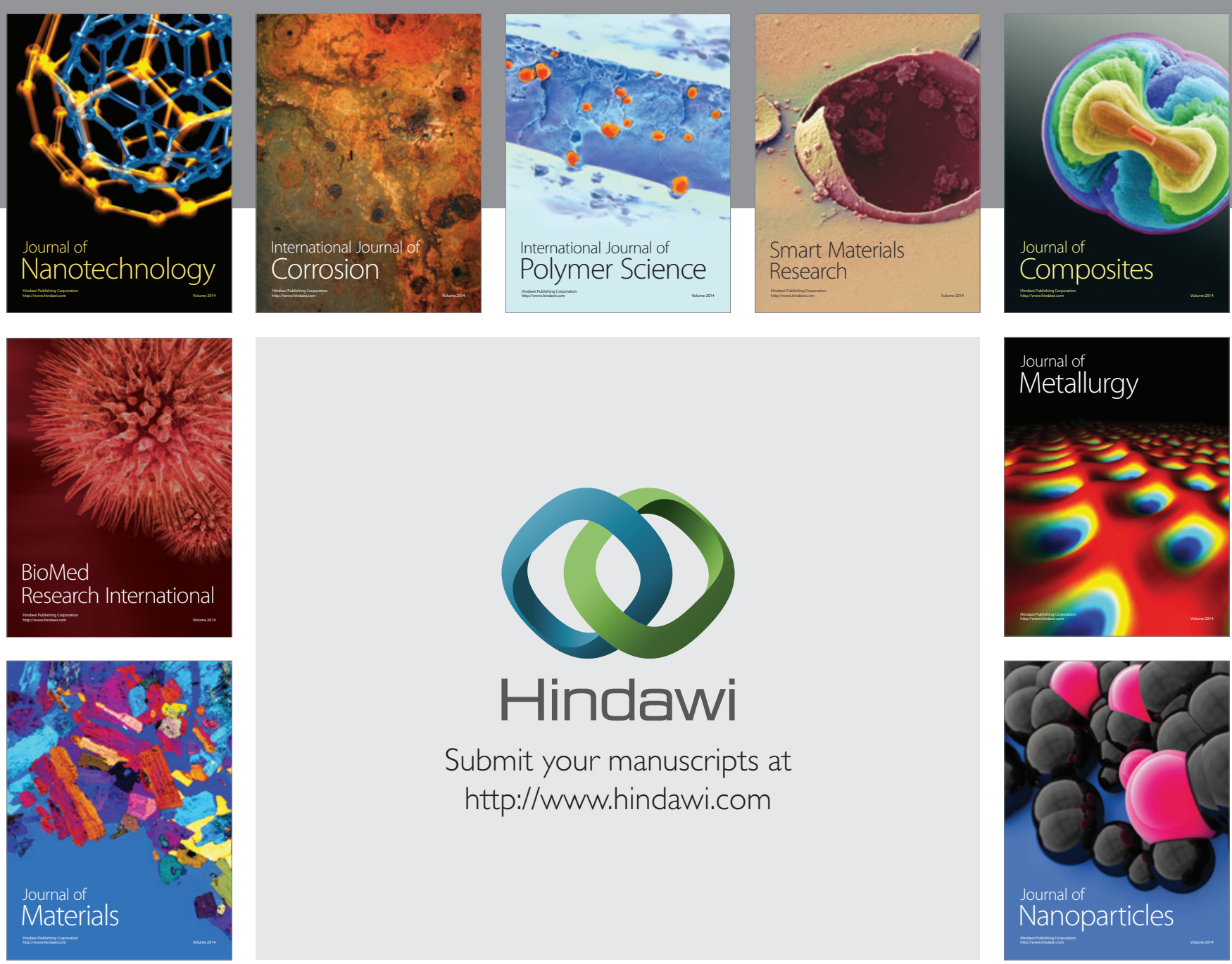

Submit your manuscripts at http://www.hindawi.com
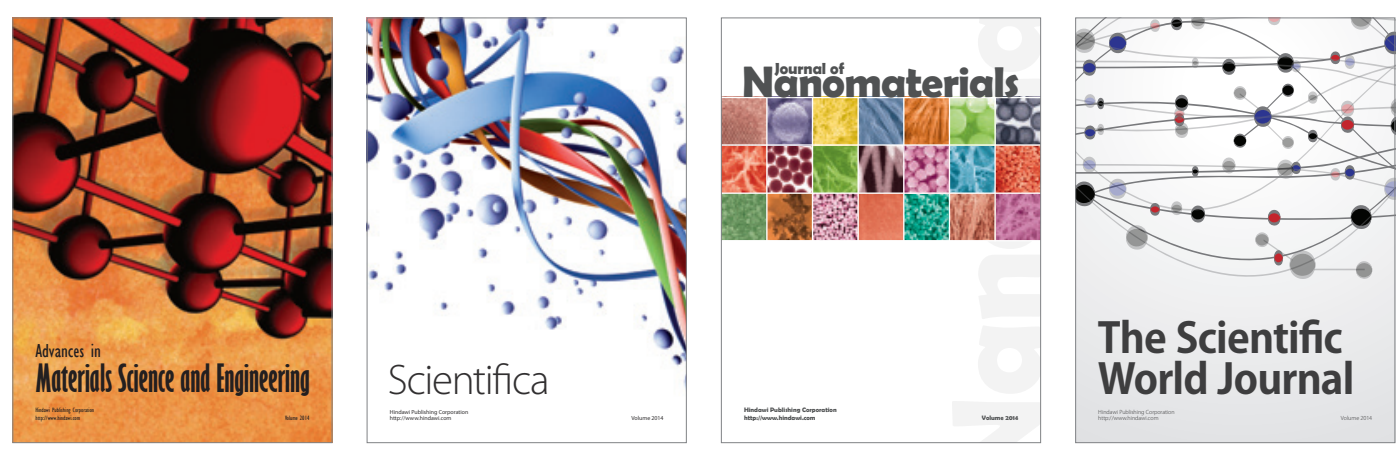

\section{The Scientific World Journal}
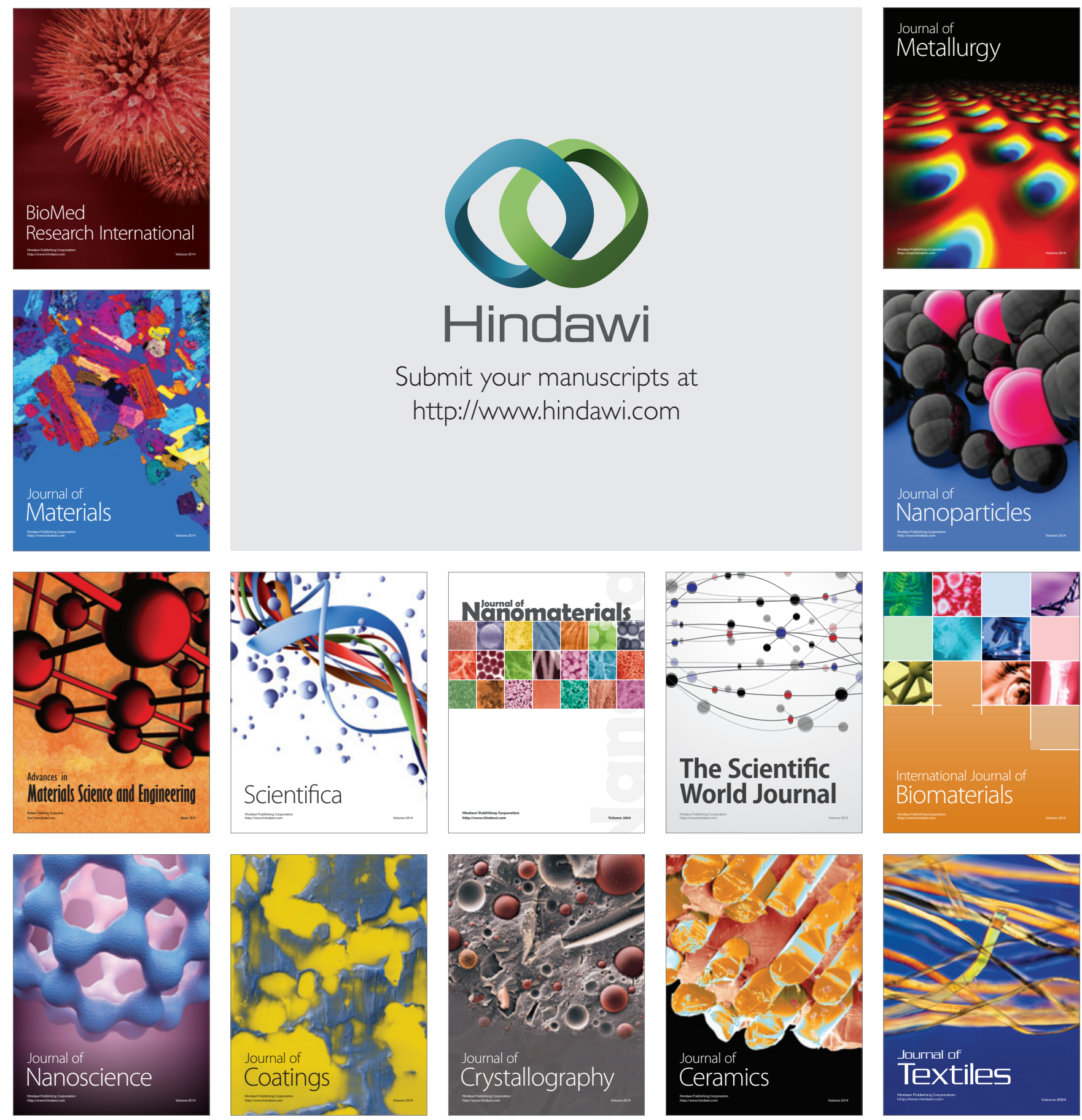